\title{
New weapon in fighting atherosclerosis: Effective inhibition of circulating low density lipoprotein cholesterol
}

\author{
Ginter $\mathrm{E}^{1}$, Simko $\mathrm{V}^{2}$ \\ Slovak Medical University, Bratislava, Slovakia. ginter.emil@mail.t-com.sk
}

\begin{abstract}
Last but not least a warning. Cholesterol is not only a pathogenic metabolite, it is an essential biological component. Indiscriminate cholesterol lowering may be inappropriate and harmful. The more effective is a weapon, the higher is the potential for a collateral damage (Fig. 1, Ref. 10). Text in PDF www.elis.sk. Key words: atherosclerosis, low density lipoprotein cholesterol (LDL C), LDL receptor (LDL- R), PCSK9, AMG 145 , statins.
\end{abstract}

Lowering low density lipoprotein cholesterol (LDL-C) is a cornerstone for the prevention of heart disease. The recent review published in BLL (1) described various methods of cholesterol management. Statins are the first line agents, being effective in reducing LDL-C. However, many patients are unable to achieve the optimal target lipid level despite an intensive statin therapy. This resulted in a strong impetus to develop novel pharmacological agents that would further decrease LDL-C in patients not adequately responding to statins.

Recent research, addressed in this review, revealed a novel pathways for reduction of LDL. Big step forward in the management of atherosclerosis is the discovery of an LDL receptor (LDL$\mathrm{R})$. This discovery was appropriately awarded a Nobel prize. LDL$\mathrm{R}$ is a cell-surface mosaic protein that mediates the endocytosis of cholesterol-rich LDL. Activity of this receptor determines the level of circulating LDL-C.

The next essential step was the identification of a factor blocking the LDL-R, a proprotein convertase subtilisin-like type 9 (PCSK9) (2). This is an extracellular protein expressed primarily in the liver. A secreted form of PCKS9 binds directly to the LDL-R and results in lysosomal degradation of LDL-R (Fig. 1). This outcome interferes with recycling of LDL-R back to the cell surface. The result is a decline of LDL-R in cell membranes, with lower binding of LDL and with an unfavorably increased amount of circulating LDL in blood vessels. Importantly, inhibition of PCSK9 is followed by an uninterrupted recycling of LDL (Fig. 1 on the right).

Genetic studies mapped PCSK9 along with LDL-R to elucidate the cause of autosomal dominant hypercholesterolemia (3). Gain-of-function mutations increased plasma levels of LDL-C while loss-of-function mutations which interfere with folding or

${ }^{1}$ Slovak Medical University, Bratislava, Slovakia (emeritus), and ${ }^{2}$ State University of New York, Downstate Medical Center at Brooklyn, USA

Address for correspondence: E. Ginter, RND, DSc, Racianska 17, SK83102 Bratislava, Slovakia.

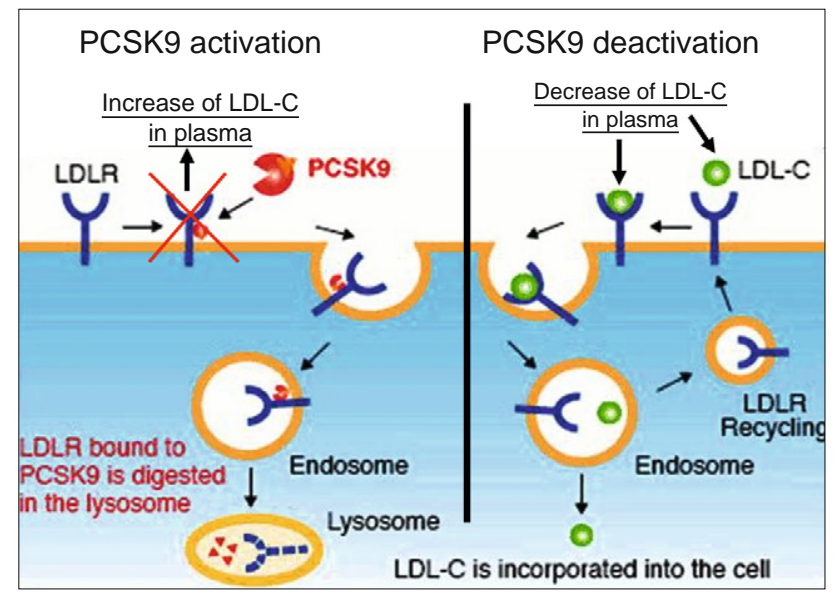

Fig. 1. PCSK9 binds directly to the LDL-R, results in degradation of the LDL receptor and increase of circulating LDL particles. Inhibition of PCSK9 by AMG 145 normalized the situation. Partly according to MBL Life Science 2012.

secretion of PCSK9, led to a reduction of plasma levels of LDL $\mathrm{C}$, along with a decrease in the risk of atherosclerosis. Statins are reported to increase the level of circulating PCSK9 (4).

This suggested the possibility that inhibition or decrease of PCSK9 may potentiate the efficacy of cholesterol lowering medications. Intensive research aimed at inhibition of PCSK9 resulted in discovery of AMG 145 (5). This compound is a fully human monoclonal immunoglobulin G2 antibody that binds specifically to human PCSK9 and inhibits its interaction with the LDL-R (6). Clinical development programs for monoclonal antibodies against PCSK9 have advanced rapidly with AMG 145. They clearly demonstrated substantial reductions in LDL-C levels in patients on AMG 145, when receiving diet alone, when on low, moderate or high doses of statins and also both in heterozygous familial hypercholesterolemia and nonfamilial hypercholesterolemia subjects ( 7 , 8). AMG 145 administered subcutaneously every 4 weeks yielded 


\section{$741-742$}

rapid and substantial reductions in LDL-C in heterozygous familial hypercholesterolemia patients despite intensive statin use, with minimal adverse events and good tolerability. Larger and much longer trials are now in progress to assess the long-term tolerability, safety, and impact on cardiovascular disease events of these very effective LDL-C lowering compounds. The results to date indicate that the AMG 145 appears safe, well-tolerated, and profoundly lowers LDL-C levels. This new compound is expected to meet an important clinical need for patients unable to achieve adequate lowering of LDL-C when on currently available therapies.

Last but not least a warning. Cholesterol is not only a pathogenic metabolite, it is an essential biological component. Indiscriminate cholesterol lowering may be inappropriate and harmful $(9,10)$. The more effective is a weapon, the higher is the potential for a collateral damage.

\section{References}

1. Ginter E, Simko V. Update on medical management of dyslipidemia and atherosclerosis. Bratisl Lek Listy 2013; 114 (5): 262-268.

2. Abifadel M, Varret M, Rabès JP et al. Mutations in PCSK9 cause autosomal dominant hypercholesterolemia. Nat Genet 2003; 34 (2): 154-156.
3. Berge KE, Ose L, Leren TP. Missense mutations in the PCSK9 gene are associated with hypocholesterolemia and possibly increased response to statin therapy. Arterioscler Thromb Vasc Biol 2006; 26 (5): 1094-1100.

4. Careskey HE, Davis RA, Alborn WE et al. Atorvastatin increases human serum levels of proprotein convertase subtilisin/kexin type 9. J. Lipid Res 2008; 49: 394-398.

5. Chan J, Piper DE, Q Cao Q et al. A PCSK9 neutralizing antibody reduces serum cholesterol in mice and nonhuman primates. Proc Natl Acad Sci 2009; 106: 9820-9825.

6. Stein EA, Swergold GD. Potential of proprotein convertase subtilisin/ kexin type 9 based therapeutics. Curr Atheroscler Rep 2013; 15 (3): 310.

7. Sullivan D, Olsson AG, Scott R et al. Effect of a monoclonal antibody to PCSK9 on low-density lipoprotein cholesterol levels in statin-intolerant patients: the GAUSS randomized trial. JAMA 2012; 308 (23): 2497-2506.

8. Davidson MH. Emerging low-density lipoprotein therapies: Targeting PCSK9 for low-density lipoprotein reduction. J Clin Lipidol 2013; 7 (Suppl 3): S11-15.

9. Saito N, Sairenchi T, Irie F et al. Low serum LDL cholesterol levels are associated with elevated mortality from liver cancer in Japan: the Ibaraki Prefectural health study. Tohoku J Exp Med 2013; 229: 203-211.

10. Simko V, Ginter E. Understanding cholesterol: high is bad but too low may also be risky. Bratisl Lek Listy 2014; 115 (2): 59-65.

Received June 8, 2013. Accepted August 18, 2014. 